\title{
On quadratic character twists of Hecke $L$-functions attached to cusp forms of varying weights at the central point
}

\author{
by \\ Winfried Kohnen (Heidelberg) and Jyoti Sengupta (Bombay)
}

1. Introduction. Let $2 k$ be a positive integer divisible by 4 . In [13] the second named author showed that the central critical values of Hecke $L$-functions $L(f, s)(s \in \mathbb{C})$ of cuspidal normalized Hecke eigenforms $f$ of weight $2 k$ with respect to $\mathrm{SL}_{2}(\mathbb{Z})$ on average satisfy an analogue of the Lindelöf hypothesis when the weight varies, i.e. one has

$$
\sum_{f \in \mathcal{F}_{2 k}} L(f, k) \ll_{\varepsilon} k^{1+\varepsilon} \quad(k \rightarrow \infty)
$$

for any $\varepsilon>0$, where $\mathcal{F}_{2 k}$ is the set of normalized cuspidal Hecke eigenforms of weight $2 k$ and the constant implied in $\ll$ only depends on $\varepsilon$ and is effective.

It is also proved in [13] that if one assumes a corresponding Lindelöf hypothesis in weight aspect for each individual $f$, i.e. for any $\varepsilon>0$ one has

$$
L(f, k) \ll_{\varepsilon} k^{\varepsilon} \quad\left(k \rightarrow \infty, f \in \mathcal{F}_{2 k}\right)
$$

(which being optimistic is suggested by (1)), then

$$
\#\left\{f \in \mathcal{F}_{2 k} \mid L(f, k) \neq 0\right\} \gg_{\varepsilon} k^{1-\varepsilon} \quad(k \rightarrow \infty)
$$

for all $\varepsilon>0$. If the constant implied in $\ll$ is effective, then also the one implied in $\gg$ is effective.

Note that one actually expects that $L(f, k) \neq 0$ for all $f \in \mathcal{F}_{2 k}$ and all $k$; for more information on this cf. [2]. The latter has been numerically checked for all even $k \leq 250$ [2]. According to [9], (2) is true with $\varepsilon=1 / 2$.

The main ingredient of the proof of the above two assertions in [13] is an estimate from above and below for the Petersson norm $\|f\|$ in weight aspect due to Iwaniec [8] and Hoffstein-Lockart [7], Goldfeld-Hoffstein-Lieman [6], respectively. The other ingredient is a formula (when specialized to the case

2000 Mathematics Subject Classification: Primary 11F46. 
$s=k)$ due to the first named author which expresses

$$
\sum_{f \in \mathcal{F}_{2 k}} \frac{L(f, s)}{\|f\|^{2}} \quad(1<\operatorname{Re}(s)<2 k-1)
$$

in terms of an infinite sum of hypergeometric functions [11].

The purpose of this note is to generalize the above two results to the case of a twist of $L(f, s)$ by a quadratic character $\left(\frac{D}{}\right)$ where $D$ is a fundamental discriminant and $k$ is an arbitrary positive integer with $(-1)^{k} D>0$.

Except for again exploiting the results of [6-8], the proof we shall give for general $D$ is different from that given in [13] for the case $D=1$. In fact, we shall use Waldspurger's result relating the twisted central critical values to squares of Fourier coefficients of modular forms of half-integral weight in the more explicit version for level 1 given in [12], together with some simple estimates for Fourier coefficients of Poincaré series of half-integral weight.

Remark. Probably our results can also be proved by properly modifying the methods developed by Duke [4]. However, we are not aware of any workout of this in the literature.

2. Statement of result. For $k$ an integer $\geq 6$ we denote by $S_{2 k}$ the space of cusp forms of weight $2 k$ with respect to $\Gamma_{1}:=\mathrm{SL}_{2}(\mathbb{Z})$. If $f \in S_{2 k}$ and $D$ is a fundamental discriminant, we denote by $L(f, D, s)(s \in \mathbb{C})$ the $L$ function of $f$ twisted with the quadratic character $(\underline{D})$ of the field extension $\mathbb{Q}(\sqrt{D}) / \mathbb{Q}$, defined by analytic continuation of the series

$$
\sum_{n \geq 1}\left(\frac{D}{n}\right) a(n) n^{-s} \quad(\operatorname{Re}(s) \gg 0 ; a(n)=n \text {th Fourier coefficient of } f) .
$$

Recall that $L(f, D, s)$ has an analytic continuation to $\mathbb{C}$ and satisfies a functional equation under $s \mapsto 2 k-s$ with root number $\left(\frac{D}{-1}\right)(-1)^{k}$. In particular, $L(f, D, k)=0$ for $(-1)^{k} D<0$.

As before we let $\mathcal{F}_{2 k}$ be the set of normalized Hecke eigenforms in $S_{2 k}$.

Theorem 1. Let D be a fundamental discriminant. Then

$$
\sum_{f \in \mathcal{F}_{2 k}} L(f, D, k) \ll_{\varepsilon, D} k^{1+\varepsilon} \quad\left(k \rightarrow \infty,(-1)^{k} D>0\right)
$$

where the constant implied in $\ll$ depends only on $\varepsilon$ and $D$ and is effective.

Theorem 2. Let $D$ be a fundamental discriminant. Let $0<\varepsilon<1$ be fixed and suppose that

$$
L(f, D, k) \ll_{\varepsilon, D} k^{\varepsilon} \quad\left(f \in \mathcal{F}_{2 k}, k \rightarrow \infty,(-1)^{k} D>0\right)
$$


with an effective constant implied in $\ll$. Then

$$
\#\left\{f \in \mathcal{F}_{2 k} \mid L(f, D, k) \neq 0\right\} \gg_{\varepsilon, D} \frac{k^{1-\varepsilon}}{\log k} \quad\left(k \rightarrow \infty,(-1)^{k} D>0\right)
$$

where the constant implied in $\gg$ is effective.

3. Proofs. Let $f \in \mathcal{F}_{2 k}$. We denote by

$$
\|f\|^{2}=\int_{\Gamma_{1} \backslash \mathcal{H}}|f(z)|^{2} y^{2 k-2} d x d y \quad(\mathcal{H}=\text { upper half-plane, } z=x+i y)
$$

the Petersson norm of $f$.

Let $F$ be the automorphic form on $\mathrm{GL}_{3}$ which is the adjoint square lift of $f$ and let $L_{\mathrm{St}}(F, s)(s \in \mathbb{C})$ be its standard zeta function, so $L_{\mathrm{St}}(F, s)$ is also the symmetric square $L$-function of $f$ (see [5]; the $L$-functions here are normalized to have functional equations under $s \mapsto 1-s)$. One then has

$$
\frac{1}{\log (2 k+1)} \ll L_{\mathrm{St}}(F, 1) \ll_{\varepsilon} k^{\varepsilon}
$$

for any $\varepsilon>0$ where the constant implied in the lower bound is absolute and all the constants implied in $\ll$ are effective. The upper bound inequality was proved in [8] and the lower bound inequality in $[6,7]$. Note that in the quoted papers the corresponding estimates were given in the context of Maass wave forms (with $2 k$ replaced by the corresponding eigenvalue $\lambda$ under the Laplace operator), but that the arguments carry over to the holomorphic case (cf. [7, p. 164] and [3, p. 1183]; cf. also [13]).

Since the symmetric square $L$-function of $f$ up to multiplication with a Riemann zeta function is the Rankin zeta function of $f$ and the latter has a simple pole at $s=1$ with residue essentially equal to $\|f\|^{2}$, we see that (3) actually gives bounds for $\|f\|$; working out the constants one finds that

$$
\frac{\Gamma(2 k)}{(4 \pi)^{2 k} \log (2 k+1)} \ll\|f\|^{2} \ll_{\varepsilon} \frac{\Gamma(2 k)}{(4 \pi)^{2 k}} k^{\varepsilon}
$$

for any $\varepsilon>0$ (cf. [13]). From (4) it follows that

$$
\begin{aligned}
\frac{\Gamma(2 k)}{(4 \pi)^{2 k} \log (2 k+1)} \sum_{f \in \mathcal{F}_{2 k}} \frac{L(f, D, k)}{\|f\|^{2}} & \ll \sum_{f \in \mathcal{F}_{2 k}} L(f, D, k) \\
& \ll_{\varepsilon} \frac{\Gamma(2 k)}{(4 \pi)^{2 k}} k^{\varepsilon} \sum_{f \in \mathcal{F}_{2 k}} \frac{L(f, D, k)}{\|f\|^{2}} .
\end{aligned}
$$
to

Denote by $S_{k+1 / 2}^{+}$the space of cusp forms of weight $k+1 / 2$ with respect

$$
\Gamma_{0}(4)=\left\{\left(\begin{array}{ll}
a & b \\
c & d
\end{array}\right) \in \Gamma_{1} \mid c \equiv 0(\bmod 4)\right\}
$$


with $n$th Fourier coefficients vanishing unless $(-1)^{k} n \equiv 0,1(\bmod 4)$, equipped with the normalized Petersson scalar product

$$
\langle g, h\rangle=\frac{1}{6} \int_{\Gamma_{0}(4) \backslash \mathcal{H}} g(z) \overline{h(z)} y^{k-3 / 2} d x d y \quad(z=x+i y) .
$$

Then according to [12] one has

$$
\frac{L(f, D, k)}{\|f\|^{2}}=\frac{\pi^{k}}{\Gamma(k)}|D|^{1 / 2-k} \frac{c(|D|)^{2}}{\|g\|^{2}}
$$

where $g \in S_{k+1 / 2}^{+}$is a Hecke eigenform with real Fourier coefficients corresponding to $f$ under the Shimura correspondence and $c(|D|)$ is the $|D|$ th Fourier coefficient of $g$. (This is a more explicit version of Waldspurger's result in the special case of level 1; note that the explicit knowledge of the constant of proportionality is very important for our purposes here.)

Let $P_{k, D}$ be the $|D|$ th Poincaré series in $S_{k+1 / 2}^{+}$characterized by

$$
\left\langle h, P_{k, D}\right\rangle=\frac{1}{6} \cdot \frac{\Gamma(k-1 / 2)}{(4 \pi|D|)^{k-1 / 2}} c_{h}(|D|)
$$

for all $h \in S_{k+1 / 2}^{+}$where $c_{h}(|D|)$ denotes the $|D|$ th Fourier coefficient of $h$. We write $P_{k, D}$ in terms of a basis $\{g\}$ of Hecke eigenforms corresponding to the basis $\mathcal{F}_{2 k}$ and take $|D|$ th Fourier coefficients. Using the implied expression for the $|D|$ th Fourier coefficient $p_{k, D}(|D|)$ of $P_{k, D}$ we find after inserting (6) into (5) that

$$
c_{k} \cdot \frac{1}{\log (2 k+1)} \cdot p_{k, D}(|D|) \ll \sum_{f \in \mathcal{F}_{2 k}} L(f, D, k) \ll_{\varepsilon} c_{k} \cdot k^{\varepsilon} \cdot p_{k, D}(|D|)
$$

where

$$
c_{k}:=\frac{\Gamma(2 k)}{\Gamma(k) \Gamma(k-1 / 2) 2^{2 k-1}}=\frac{1}{\sqrt{\pi}}\left(k-\frac{1}{2}\right) .
$$

The Fourier coefficients of $P_{k, D}$ were computed in [10]. In particular, one has

$$
\begin{aligned}
p_{k, D} & (|D|) \\
= & \frac{2}{3}\left(1+(-1)^{[(k+1) / 2]} \pi \sqrt{2} \sum_{c \geq 1} \frac{1}{4 c} H_{c}(|D|,|D|) J_{k-1 / 2}\left(\frac{\pi|D|}{c}\right)\right)
\end{aligned}
$$

where

$$
\begin{aligned}
& H_{c}(|D|,|D|) \\
& =\left(1-(-1)^{k} i\right)\left(1+\left(\frac{4}{c}\right)\right) \sum_{d(4 c)^{*}}\left(\frac{4 c}{d}\right)\left(\frac{-4}{d}\right)^{k+1 / 2} e^{2 \pi i|D|\left(d+d^{-1}\right) /(4 c)}
\end{aligned}
$$


is a generalized Kloosterman sum and $J_{k-1 / 2}$ is the Bessel function of order $k-1 / 2$. In (9) the summation is over a primitive residue system modulo $4 c, d^{-1}$ denotes an integer with $d^{-1} d \equiv 1(\bmod 4 c),(\vdots)$ is the generalized Jacobi-Kronecker symbol and $\left(\frac{-4}{d}\right)^{1 / 2}$ is equal to 1 or $i$ according as $d \equiv 1$ $(\bmod 4)$ or $d \equiv 3(\bmod 4)$, respectively.

The Poisson integral representation

$$
\begin{aligned}
J_{k-1 / 2}(z)=\sqrt{\frac{2}{\pi}} \cdot \frac{z^{k-1 / 2}}{2^{k} \Gamma(k)} \int_{0}^{\pi} \cos (z \cos \theta) \sin ^{2 k-1} \theta d \theta \\
\quad\left(z \neq 0,-\pi / 2<\arg z^{1 / 2} \leq \pi / 2\right)
\end{aligned}
$$

$[1$, formula 10.1.13] shows that

$$
\left|J_{k-1 / 2}(x)\right| \leq \sqrt{\frac{2}{\pi}} \cdot \frac{x^{k-1 / 2}}{2^{k} \Gamma(k)}
$$

for positive real $x$.

We split up the sum in (8) into the sum of the finitely many terms with $c<\pi|D|$ and the sum over the terms with $c>\pi|D|$. Using the trivial bound

$$
\left|H_{c}(|D|,|D|)\right| \leq \sqrt{2} \cdot 8 c
$$

we then immediately deduce that

$$
p_{k, D}(|D|) \ll_{D} 1 \quad(k \rightarrow \infty)
$$

and

$$
p_{k, D}(|D|) \gg_{D, \delta} \frac{2}{3}-\delta \quad(k \rightarrow \infty)
$$

for any fixed $\delta>0$.

Taking into account the value of $c_{k}$, we find that (10) and the second inequality in (7) imply the assertion of Theorem 1. Likewise (11) and the first inequality in (7) imply Theorem 2.

\section{References}

[1] M. Abramowitz and I. Stegun, Handbook of Mathematical Functions, Dover, New York, 1965.

[2] J. B. Conrey and D. Farmer, Hecke operators and the non-vanishing of L-functions, in: Topics in Number Theory, S. D. Ahlgren, G. E. Andrews and K. Ono (eds.), Kluwer, Dordrecht, 1999, 143-150.

[3] J. B. Conrey and H. Iwaniec, The cubic moment of central values of automorphic L-functions, Ann. of Math. 151 (2000), 1175-1216.

[4] W. Duke, The critical order of vanishing of automorphic L-functions with large level, Invent. Math. 119 (1995), 165-174.

[5] S. Gelbart and H. Jacquet, A relation between automorphic representations of $G L_{2}$ and $G L_{3}$, Ann. Sci. École Norm. Sup. (4) 11 (1978), 471-552. 
[6] D. Goldfeld, J. Hoffstein and D. Lieman, An effective zero-free region, Ann. of Math. 140, appendix to the paper quoted as [7] below.

[7] J. Hoffstein and P. Lockart, Coefficients of Maass forms and the Siegel zero, Ann. of Math. 140 (1994), 161-181.

[8] H. Iwaniec, Small eigenvalues of Laplacian for $\Gamma_{0}(N)$, Acta Arith. 56 (1990), 65-82.

[9] W. Kohnen, Modular forms of half-integral weight on $\Gamma_{0}(4)$, Math. Ann. 248 (1980), 249-266.

[10] - Fourier coefficients of modular forms of half-integral weight, ibid. 271 (1985), $237-268$.

[11] - Non-vanishing of Hecke L-functions associated to cusp forms inside the critical strip, J. Number Theory 67 (1997), 182-189.

[12] W. Kohnen and D. Zagier, Values of L-series of modular forms at the center of the critical strip, Invent. Math. 64 (1981), 175-198.

[13] J. Sengupta, The central critical values of automorphic L-functions, C. R. Math. Acad. Sci. Soc. R. Can. 22 (2000), no. 2, 82-85.

Mathematisches Institut, INF 288

Universität Heidelberg

D-69120 Heidelberg, Germany

E-mail: winfried@mathi.uni-heidelberg.de
School of Mathematics

T.I.F.R.

Homi Bhabha Road

400005 Bombay, India

E-mail: sengupta@math.tifr.res.in 